\title{
Emergency department point-of-care biomarkers and day 90 functional outcome in spontaneous intracerebral hemorrhage: A single-center pilot study
}

\author{
EUGENIA-MARIA MUREȘAN ${ }^{1 *}$, ADELA GOLEA $^{2 *}$, ȘTEFAN CRISTIAN VESA $^{3}$, \\ MANUELA LENGHEL ${ }^{4}$, CSABA CSUTAK $^{4}$ and LĂCRĂMIOARA PERJU-DUMBRAVĂ ${ }^{1}$
}

\begin{abstract}
${ }^{1}$ Department of Neurosciences, Faculty of Medicine, 'Iuliu Hațieganu' University of Medicine and Pharmacy, 400012 Cluj-Napoca; ${ }^{2}$ Department of Surgery, Emergency Medicine Discipline, 'Iuliu Hațieganu' University of Medicine and Pharmacy, 400006 Cluj-Napoca; ${ }^{3}$ Department of Pharmacology, Toxicology and Clinical Pharmacology, Faculty of Medicine, 'Iuliu Haţieganu' University of Medicine and Pharmacy, 400349 Cluj-Napoca; ${ }^{4}$ Department of Surgical Specialties, Radiology Discipline, 'Iuliu Hațieganu', University of Medicine and Pharmacy, 400162 Cluj-Napoca, Romania
\end{abstract}

Received November 2, 2021; Accepted December 1, 2021

DOI: $10.3892 / \mathrm{etm} .2022 .11123$

\begin{abstract}
Spontaneous intracerebral hemorrhage (sICH) results in high morbidity and mortality rates, thus identifying strategies for timely prognosis and treatment is important. The present study aimed to analyze the relationship between emergency department point-of-care (POC) blood biomarkers and day 90 functional outcome (FO) in patients with acute $(<8 \mathrm{~h})$ sICH. On-site POC determinations, including complete blood count, glucose, cardiac troponin I, D-dimer and C-reactive protein, and derived inflammatory indexes were performed for a cohort of 35 patients. The primary endpoint was a favorable day 90 FO (modified Rankin Score $\leq 3$ ). Secondary endpoints included early neurological worsening (ENW), day 7/discharge neurological impairment, day 90 independence assessment (Barthel Index <60), hematoma enlargement and perihematomal edema (PHE) growth. A favorable three-month FO was reported in $16(46 \%)$ participants. Older age, previous history of ischemic stroke and initial imagistic parameters, including intraventricular hemorrhage, enlarged contralateral ventricle and cerebral atrophy, significantly predicted an unfavorable FO. The admission D-dimer similarly predicted day $90 \mathrm{FO}$ and the independence status, along with ENW and a more severe day $7 /$ discharge neurological status. The D-dimer also
\end{abstract}

Correspondence to: Dr Ștefan Cristian Vesa, Department of Pharmacology, Toxicology and Clinical Pharmacology, Faculty of Medicine, 'Iuliu Haţieganu' University of Medicine and Pharmacy, Strada Gheorghe Marinescu 23, 400349 Cluj-Napoca, Romania

E-mail: stefanvesa@gmail.com

${ }^{*}$ Contributed equally

Key words: spontaneous intracerebral hemorrhage, point-of-care testing, biomarkers, D-dimer, functional outcome, emergency department correlated with the initial neurological status and PHE. PHE growth correlated with granulocytes, systemic immune-inflammation index and glycemia. The results suggested that a lower admission D-dimer could indicate an improved day 90 FO of patients with sICH, while also anticipating the development of PHE growth and ENW.

\section{Introduction}

Spontaneous intracerebral hemorrhage (sICH) has a disproportionate socioeconomic impact considering its low incidence rate (26\% of all incident strokes in 2017, with higher prevalence rates in East European countries) (1). An aging population and repeated unsuccessful research endeavors for curative treatment contribute to its high mortality and morbidity, resulting in a 1-month case fatality of $40 \%$ with only $12 \%$ of patients regaining long-term functional independence (2). At present, the focus is on improving early outcome prediction to individualize patient management, and to also identify individuals at risk before sICH occurs, as to date, no reliable premonitory onset markers have been determined. Therefore, biomarker testing is an area of interest, as it is minimally invasive, low cost and could potentially enable accurate risk stratification and outcome estimation. Routinely, the standard hematologic evaluation consists of complete blood count (CBC), including platelet (PLT) count, coagulation profile and serum glucose $(3,4)$. As sICH is a time-sensitive condition, readily available point-of-care (POC) devices reduce delays and facilitate prompt management.

Over the past decade, the role of inflammation in $\mathrm{sICH}$ progression and neurological impairment has been further clarified $(5,6)$, and inflammatory biomarkers are currently regarded as potential prognostication tools. CBC upon admission, which includes hemoglobin ( $\mathrm{Hb})$, red blood cells and their distribution width (RDW), and derived inflammatory indexes, such as neutrophils-to-lymphocytes ratio (NLR), lymphocytes-to-monocytes ratio (LMR), platelets-to-lymphocytes ratio (PLR) and C-reactive protein (CRP), have been 
associated with mortality (5,7-11). Moreover, early neurological worsening (ENW) (12), hematoma volume expansion and the expansion of the surrounding edema $(5,13,14)$, and day 90 functional outcome (FO) $(6,13,15-19)$ have also been reported to be associated with mortality. Furthermore, systemic immune-inflammation index (SII) has been recently reported as a relevant predictor of poor hospital discharge outcome (20).

Leukocyte count has been consistently associated with larger ICH volumes (5), but no consensus has been reached as to ICH progression, infection risk and mortality (11). Higher admission neutrophils have been associated with larger baseline volumes $(21)$, mortality $(10,22,23)$ and morbidity $(10,23)$. With regard to monocyte (MON) count, increased admission levels are associated with poor outcome and mortality (5), but not with ICH volume (21), as MON are thought to contribute to secondary injury (13). Admission lymphopenia has been correlated with higher stroke severity, larger baseline hematoma volume and intraventricular extension, along with infection risk and 3-month mortality rate (5). NLR and LMR mirror post-ICH proinflammation and immunosuppression (12), as higher NLR has reflected larger baseline volumes, stroke severity, severe perihematomal edema (PHE) growth and poor 3-month outcome (5), and lower LMR has indicated neurologic deterioration and day 90 mortality (12).

CRP has been significantly linked with hematoma growth (HG), ENW, mortality and 3-month outcome $(5,7,9,24)$. Its early presence at the hemorrhagic site could be due to local synthesis or transformation of the circulating liver-synthetized pentameric form (24).

Regarding $\mathrm{Hb}$, anemia is associated with larger $\mathrm{ICH}$ volumes (25), increased HG (26) and worse outcomes (26-28). RDW is another inexpensive, automatically generated hematology parameter that is impacted by inflammation and is currently associated with day 30 FO (29).

Moreover, stroke is considered a systemic condition that induces cardiac, lung and immune dysfunctions $(5,30)$; therefore, cardiac biomarkers, such as troponin I (cTnI), have been linked to stroke severity $(31)$, in-hospital mortality $(30,32)$ and unfavorable outcomes $(33,34)$. On the other hand, D-dimer levels have been associated with an increased risk $(35,36)$ and severity (37) of hemorrhagic stroke, and an increased hematoma volume (37), although it has not been proved sufficiently accurate for molecular stroke diagnosis (38). Furthermore, admission hyperglycemia has also been related to mortality $(6,8)$ and day 90 FO (6).

The emergency department (ED) provides a unique opportunity for POC testing, both for standard and additional biomarkers (e.g., cTnI, D-dimer and CRP). When addressing time-sensitive conditions such as sICH, targeted escalation of the standard protocol could benefit these hyperacute patients. The contribution of additional POC testing could enable early risk stratification strategies to be identified and facilitate improvements in outcomes for patients with sICH. Nevertheless, information about the applicability of POC testing on cerebral hemorrhage is scarce.

The present study aimed to assess the predictive role of ED-based POC biomarkers (standard and additional) and derived inflammatory indexes on day 90 FO in patients with acute sICH.

\section{Materials and methods}

Patient recruitment. The design and enrolment processes of this prospective, single-center, ED-based pilot study have been previously published (39). To summarize, adult patients presenting with acute $\mathrm{sICH}(<8 \mathrm{~h}$ from onset) to the ED of the County Emergency Hospital (Cluj-Napoca, Romania) were recruited over 18 months (December 2017 to June 2018) provided that Glasgow Coma Scale (GCS) was $\geq 8$ and no exclusion criteria were met. The exclusion criteria were as follows: Identifiable secondary ICH causes, thromboembolic/ischemic disease, seizures, severe pre-ICH disability [modified Rankin Scale $(m R S) \geq 4$ ], coagulopathy, treatment with heparin, low-molecular-weight heparin, glycoprotein IIb/IIIa antagonists or oral anticoagulants, pregnancy/breastfeeding, scheduled neurosurgical/hemostatic treatment, enrolment in other studies within the last 30 days or terminal disease. The study protocol was approved by the Institutional Review Board of the 'Iuliu Haţieganu' University of Medicine and Pharmacy Cluj-Napoca (approval no. 441/24.11.2016). The procedures and interventions in the present study were in accordance with the principles stated by the Declaration of Helsinki. All participants or legal representatives provided written informed consent.

Data sources/measurements. Demographic, clinical and laboratory data were documented upon ED admission. The routine management of patients with acute sICH in our department and the study of specific interventions are present in Fig. 1. ED-based POC whole-blood analyzers included the Fujifilm Dry-Chem NX500 biochemistry analyzer and the Swelab Alfa Plus hematology analyzer. CBC included granulocytes [GRA; composed of neutrophiles (NEU) and the largest proportion of eosinophils (EOS)] and mid-size (MID) population of cells (composed of mid-size population of MON, basophils, EOS, blasts and other immature cells). Calculated hematology indexes included the following ratios: NLR (incorporating GRA values), LMR (incorporating MID values) and PLR, alongside SII [calculated as NEU x PLT/(lymphocytes (LYM) x1,000)] and incorporating GRA results. An ED-based PathFast ${ }^{\mathrm{TM}}$ fully automatic chemiluminescence enzyme immunoassay was used to study additional biomarkers, including cTnI, D-dimer and high sensitive CRP (hs-CRP).

Patients were clinically assessed on days 2 and 7 (or on discharge). Follow-up telephone interviews on day 90 included FO (mRS) and independence on daily living activities [Barthel Index (BI)].

Diagnosis and imagistic controls were performed on a General Electric Optima 64 scanner (Cytiva). The hemorrhage volume was measured using manual segmentation with the inclusion of the entire visible lesion area. The post-processing analysis was performed on a General Electric AW Server 2.0 workstation by two independent radiologists, blinded for patient outcome.

Statistical analysis. In this analysis, the primary endpoint was day 90 FO. A favorable outcome was considered as an mRS of 0-3, whereas an mRS of 4-6 was considered as an unfavorable outcome. Secondary clinical endpoints included ENW [defined as a GCS decrease of $\leq 2$ points or a National Institute 


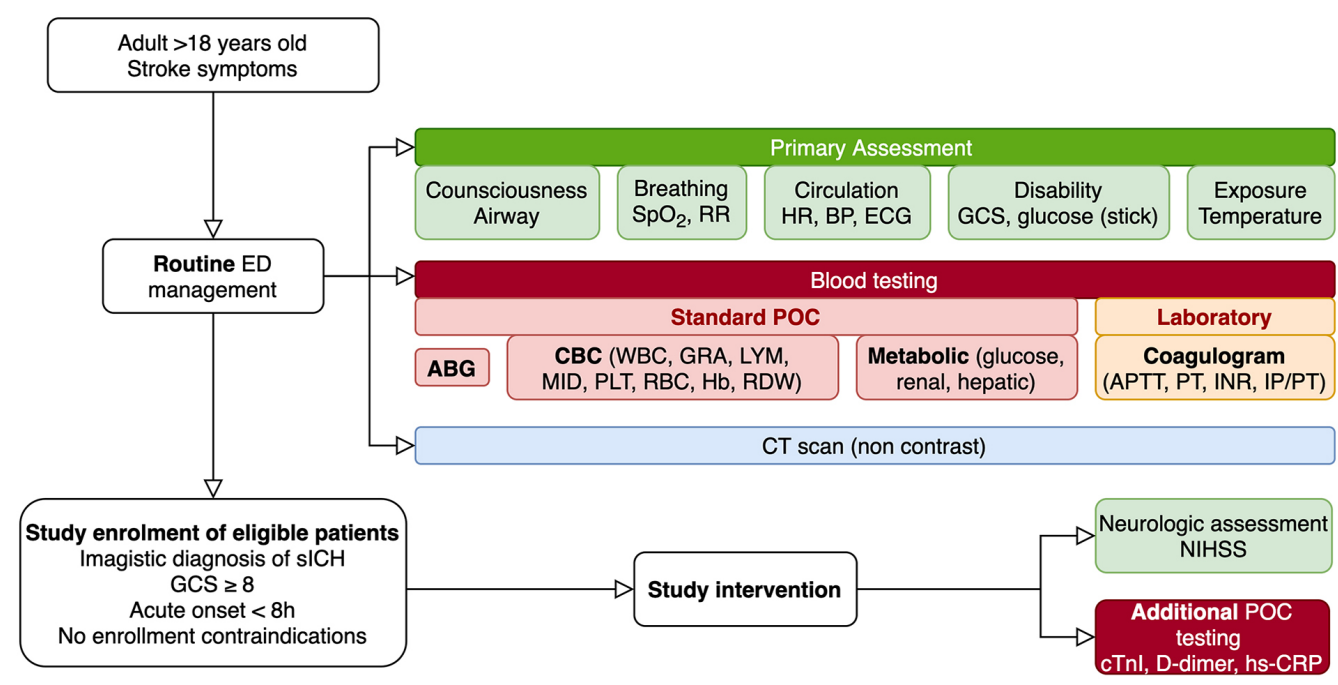

Figure 1. Routine ED baseline assessment of patients with $\mathrm{sICH}$ and study specific interventions. ED, emergency department; $\mathrm{SpO}_{2}$, peripheral oxygen saturation; RR, respiratory rate; HR, heart rate; BP, blood pressure; ECG, electrocardiogram; GCS, Glasgow Coma Scale score; POC, point-of-care; ABG, arterial blood gases; CBC, complete blood count; WBC, white blood cells; GRA, granulocytes; LYM, lymphocytes; MID, mid-cell population; PLT, platelets; RBC, red blood cells; Hb, hemoglobin; RDW, red cell distribution width; APTT, activated partial prothrombin time; PT, prothrombin time; INR, international normalized ratio; IP/PT, prothrombin index/prothrombin time; CT, computer tomography; sICH, spontaneous intracerebral hemorrhage; NIHSS, National Institute of Health Stroke Scale; cTnI, cardiac troponin I; hs-CRP, high-sensitive C reactive protein.

of Health Stroke Scale (NIHSS) increase $\geq 4$ ], day 7/discharge neurological impairment (NIHSS $\leq 15$ ), and day 90 assessment of quality of life and independence. Radiological endpoints included HG (change in baseline hematoma volume of $>33 \%$ or $>6 \mathrm{ml}$ by day 2) and PHE growth [difference in the largest PHE linear dimension between the diagnostic and control computed tomography (CT) scans].

Statistical analyses was performed using $\mathrm{MedCalc}^{\circledR}$ Statistical software (version 19.6; MedCalc Software byba). Quantitative data was assessed for normality of distribution using the Shapiro-Wilk test, and presented as the median and 25-75th percentiles. Qualitative data are presented as the frequency and percentage. Comparisons between groups were analyzed using the Mann-Whitney U test or $\chi^{2}$ test. Spearman's rho was used to assess correlations between variables. $\mathrm{P}<0.05$ was considered to indicate a statistically significant difference.

\section{Results}

A cohort of 39 patients was recruited, with 35 completing the in-hospital follow-up and $23(66 \%)$ alive on day 90 . All deaths were registered within the first month and in-hospital mortality was $23 \%$.

Baseline characteristics of the cohort according to day 90 FO are presented in Table I. Older age, previous history of ischemic stroke, and the presence of intraventricular hemorrhage (IVH), enlarged contralateral ventricle (ECV) and cerebral atrophy on the initial CT scan significantly predicted an unfortunate FO scoring on day 90 follow-up. Median baseline hematoma volume and PHE did not differ significantly between surviving [hematoma volume, $9.15 \mathrm{~cm}^{3}(6.69-22.18)$; PHE, $8.35 \mathrm{~mm}$ (6.81-11.58)] and deceased [hematoma volume, $16.83 \mathrm{~cm}^{3}$ (9.2-31.89); PHE, $\left.9.35 \mathrm{~mm}(6.50-13.25)\right]$ day 90 outcome groups ( $\mathrm{P}=0.234$ and $\mathrm{P}=0.470$, respectively).

Baseline POC biomarker values and calculated indexes according to day $90 \mathrm{mRS}$ are presented in Table II. Higher values in the unfavorable outcome group were documented for RDWa, GRA, NLR, PLR, SII, hs-CRP and D-dimer, but the differences were only significant for $\mathrm{D}$-dimer $(\mathrm{P}<0.001)$. When further considering day 90 independence on daily living activities, $\mathrm{D}$-dimer values were significantly higher $(\mathrm{P}=0.032)$ in the dependent patients [BI $<60 ; 3.610 \mu \mathrm{g} / \mathrm{ml}$ FEU $(0.900-5.010)$ vs. $0.758 \mu \mathrm{g} / \mathrm{ml}$ FEU $(0.383-0.890)]$ compared with those in the day 90 independent group. Negative correlations were documented between admission D-dimer and admission GCS (rho=-0.342; $\mathrm{P}=0.044)$ and day 90 independence status (rho $=-0.670 ; \mathrm{P}=0.001)$.

ENW was documented in 9/35 patients, with only two alive by day 90 , equally divided between outcome groups. Baseline median hematoma volume and PHE did not differ significantly ( $\mathrm{P}=0.051$ and $\mathrm{P}=0.094$, respectively) between those with [hematoma volume, $24.60 \mathrm{~cm}^{3}$ (14.16-70.00); PHE, $10.50 \mathrm{~mm}(9.23-20.63)]$ and without ENW [hematoma volume, $9.20 \mathrm{~cm}^{3}$ (5.99-21.25); PHE, $7.5 \mathrm{~mm}$ (6.00-10.40)]. All ENW patients received antibiotic treatment by day 7 (ATB 7) and a modest correlation between ENW and ATB 7 was observed (rho $=0.367, \mathrm{P}=0.033$ ). In patients who developed $\mathrm{ENW}$, white

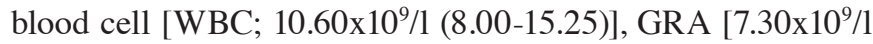
(5.95-11.60)] and D-dimer [3.73 $\mu \mathrm{g} / \mathrm{ml}$ FEU (1.22-5.01)] values were significantly higher compared with those in patients without ENW [WBC, 8.10x10 $/ 1$ (6.60-10.60); GRA, 5.30x10 $/ 1$ (3.90-7.55); D-dimer, $0.86 \mu \mathrm{g} / \mathrm{ml} \mathrm{FEU} \mathrm{(0.63-1.63)]}$ $(\mathrm{P}=0.042, \mathrm{P}=0.025$ and $\mathrm{P}=0.024$, respectively). $\mathrm{D}$-dimer was also significantly higher in the $8 / 35$ patients with a worse day 7 neurological status, defined as NIHSS score $\geq 16$ [2.01 (1.20-5.01) vs. 0.84 (0.59-1.93); $\mathrm{P}=0.017)$. By contrast, SII values were not significantly higher in either ENW group [0.45 (0.33-0.86) vs. 0.83 (0.37-1.36); $\mathrm{P}=0.265]$, nor in those patients with a worse day 7 neurological status $[0.45(0.32-1.01)$ vs. 0.49 (0.37-0.81); $\mathrm{P}=0.315]$.

The median length of hospital stay was 15 days. Two participants required neurosurgery and another one required 
Table I. Baseline clinical and imagistic characteristics.

\begin{tabular}{|c|c|c|c|}
\hline \multirow[b]{2}{*}{ Characteristic } & \multicolumn{2}{|c|}{ Day 90 FO } & \multirow[b]{2}{*}{ P-value } \\
\hline & Favorable $(n=16)$ & Unfavorable $(\mathrm{n}=19)$ & \\
\hline Median age (range), years & $62(57-68.5)$ & $75(73-81)$ & $<0.001$ \\
\hline$>70$ & $3(18.8)$ & $17(89.5)$ & $<0.001$ \\
\hline Male, n (\%) & $11(68.8)$ & $8(42.1)$ & 0.217 \\
\hline Hypertension, n (\%) & $12(75.0)$ & $15(78.9)$ & 1.000 \\
\hline$>2$ antihypertensive drugs & $7(43.8)$ & $10(52.6)$ & 0.854 \\
\hline Diabetes mellitus, n (\%) & $6(37.5)$ & $4(21.1)$ & 0.454 \\
\hline Dyslipidemia, n (\%) & $7(43.8)$ & $7(36.8)$ & 0.945 \\
\hline Statin use prior to admission, $\mathrm{n}(\%)$ & $4(25)$ & $6(31.6)$ & 0.723 \\
\hline Antiplatelet agent, n (\%) & $4(25.0)$ & $5(26.3)$ & 1.000 \\
\hline Smoking (former/active), n (\%) & $11(68.8)$ & $8(42.1)$ & 0.217 \\
\hline GCS, median (range) & $15(14 ; 15)$ & $13(12 ; 15)$ & 0.080 \\
\hline NIHSS score, median (range) & $8.5(6.2 ; 14.5)$ & $15(6 ; 21)$ & 0.288 \\
\hline Median SBP (range), mmHg & $164.5(154.5-192.5)$ & $163(147.2-174.2)$ & 0.174 \\
\hline$>170 \mathrm{mmHg}, \mathrm{n}(\%)$ & $7(43.8)$ & $8(42.1)$ & 1.000 \\
\hline Median HR (range), beats/min & $81.5(71.5-97.5)$ & $75(65-86)$ & 0.267 \\
\hline Atrial fibrillation, $\mathrm{n}(\%)$ & $1(6.2)$ & $0(0)$ & 0.457 \\
\hline Hematoma location, n (\%) & & & 0.581 \\
\hline Supra-tentorial lobar & $2(12.5)$ & $4(21.1)$ & \\
\hline Supra-tentorial deep & $14(87.5)$ & $13(68.4)$ & \\
\hline Supra-tentorial mixte & $0(0)$ & $1(5.3)$ & \\
\hline Infratentorial & $0(0)$ & $1(5.3)$ & \\
\hline Hematoma volume, $\mathrm{cm}^{3}, \mathrm{n}(\%)$ & & & 0.316 \\
\hline$<30$ & $14(87.5)$ & $14(73.7)$ & \\
\hline $30-60$ & $2(12.5)$ & $2(10.5)$ & \\
\hline$>60$ & $0(0)$ & $3(15.8)$ & \\
\hline Median perihematomal edema (range), $\mathrm{mm}$ & $9.07(7.16-28.21)$ & $21.47(6.97-32.86)$ & 0.371 \\
\hline IVH, n $(\%)$ & $1(6.2)$ & $8(42.1)$ & 0.022 \\
\hline MLS > 10 mm, n (\%) & $3(18.8)$ & $10(52.6)$ & 0.086 \\
\hline Mass effect, $\mathrm{n}(\%)$ & $12(75.0)$ & $17(89.5)$ & 0.379 \\
\hline $\mathrm{CVC}, \mathrm{n}(\%)$ & $12(75.0)$ & $16(84.2)$ & 0.677 \\
\hline $\mathrm{ECV}, \mathrm{n}(\%)$ & $1(6.2)$ & $8(42.1)$ & 0.022 \\
\hline Periventricular leucoaraiosis, n (\%) & $5(31.2)$ & $13(68.4)$ & 0.064 \\
\hline Lacunarism, n (\%) & $8(50.0)$ & $16(84.2)$ & 0.071 \\
\hline Cerebral atrophy, n (\%) & $4(25.0)$ & $16(84.2)$ & 0.001 \\
\hline Median length of hospital stay (range), days & $15(11.5-16.75)$ & $14(8-19)$ & 0.715 \\
\hline Discharge disposition & & & 0.741 \\
\hline Family care & $9(56.2)$ & $12(63.2)$ & \\
\hline Rehabilitation/lower rank hospital & $1(6.2)$ & $2(10.5)$ & \\
\hline
\end{tabular}

FO, functional outcome; mRS, modified Rankin Scale; TIA, transient ischemic attack; SBP, systolic blood pressure; HR, heart rate; GCS, Glasgow coma scale; IVH, intraventricular hemorrhage; MLS, midline shift; CVC, contralateral ventricle compression; ECV, enlarged contralateral ventricle; NIHSS, National Institute of Health Stroke Scale.

advanced intensive care unit (ICU) airway management. By day 7, medical complications occurred in 20 (57\%) participants, all of whom were undergoing antibiotic treatments. A day 90 favorable outcome was reached in only $25 \%$ patients. No significant POC biomarker differences were documented between patients with and without day 7 antibiotic treatment.
Control CT scans were performed for 29/35 participants (83\%). Only 7 controls were performed within the first $48 \mathrm{~h}$ (median time of 6 days and $13 \mathrm{~h}$ since the onset of symptoms). Any hematoma expansion occurred in $15 / 29$ participants [median $2.46 \mathrm{~cm}^{3}\left(1.78-11.18 \mathrm{~cm}^{3}\right)$ ], yet the criteria for $\mathrm{HG}$ was fulfilled in only $6(40 \%)$ patients, with 4 dying before the 
Table II. Comparison of admission POC biomarkers according to day 90 outcome.

Day 90 FO

\begin{tabular}{|c|c|c|c|}
\hline POC biomarker & Favorable $(n=16)$ & Unfavorable $(\mathrm{n}=19)$ & P-value \\
\hline $\mathrm{Hb}, \mathrm{g} / \mathrm{dl}$ & $13.85(12.75-15.07)$ & $13.40(12.70-14.80)$ & 0.417 \\
\hline $\mathrm{RBC}, \times 10^{12} / 1$ & $4.55(4.33-4.91)$ & $4.55(4.08-4.94)$ & 0.729 \\
\hline RDWa, fl & $60.30(58.13-65.38)$ & $63.20(58.20-66.40)$ & 0.943 \\
\hline WBC, $x 10^{9} / 1$ & $9.35(6.45-11.58)$ & $9.30(6.70-11.30)$ & 0.895 \\
\hline GRA, $x 10^{9} / 1$ & $5.40(3.80-8.60)$ & $6.20(4.70-8.30)$ & 0.667 \\
\hline LYM, x $10^{9} / 1$ & $2.15(1.33-2.68)$ & $1.80(1.40-2.20)$ & 0.127 \\
\hline MID, $x 10^{9} / 1$ & $0.85(0.60-1.10)$ & $0.80(0.60-1.30)$ & 0.934 \\
\hline PLT, $x 10^{9} / 1$ & $168.50(140.00-221.50)$ & $162.00(150.00-192.00)$ & 0.740 \\
\hline NLR & $2.26(1.82-3.70)$ & $3.14(2.79-5.14)$ & 0.145 \\
\hline LMR & $2.11(1.71-4.33)$ & $2.11(1.20-3.25)$ & 0.486 \\
\hline PLR & $92.94(55.2-125.00)$ & $95.21(72.5-116.48)$ & 0.655 \\
\hline SII & $0.39(0.25-1.15)$ & $0.53(0.36-0.88)$ & 0.868 \\
\hline hs-CRP, mg/l & $2.49(0.89-4.01)$ & $3.27(0.68-5.20)$ & 0.446 \\
\hline $\mathrm{cTnI}, \mathrm{ng} / \mathrm{ml}$ & $0.003(0.002-0.070)$ & $0.003(0.001-0.006)$ & 0.181 \\
\hline D-dimer, $\mu \mathrm{g} / \mathrm{ml}$ FEU & $0.75(0.38-0.89)$ & $2.31(0.92-5.01)$ & $<0.001$ \\
\hline Glucose, $\mathrm{mmol} / \mathrm{l}$ & $146(143-168)$ & $140(119-183)$ & 0.585 \\
\hline
\end{tabular}

Data are presented as the median (range). FO, functional outcome; POC, point-of-care; WBC, white blood cells; GRA, granulocytes; LYM, lymphocytes; MID, mid-cell fractions; PLT, platelets; NLR, neutrophils-to-lymphocytes ratio; LMR, lymphocytes-to-monocytes ratio; PLR, platelets-to-lymphocytes ratio; SII, systemic immune-inflammation index; Hb, hemoglobin; RBC, red blood cells; RDW, red cells distribution width; hs-CRP, high sensitive C reactive protein; cTnI, cardiac troponin I.

follow-up. A median PHE growth of $3.65 \mathrm{~mm}(1.38-8.38 \mathrm{~mm})$ was documented in 25 participants (12/19 unfavorable outcome group). Moderate or weak negative correlations were detected between POC inflammatory markers and indexes and PHE growth (rho=- $0.511, \mathrm{P}=0.005$ for $\mathrm{WBC}$; rho $=-0.548, \mathrm{P}=0.002$ for GRA; rho $=-0.373, \mathrm{P}=0.047$ for SII; rho $=-0.378, \mathrm{P}=0.043$ for glucose). D-dimer was correlated with admission PHE (rho $=0.398, \mathrm{P}=0.018$ ).

Cut-off values of the variables mostly associated with the primary outcome were calculated, namely age, admission GCS and D-dimer. As such, an unfavorable day $90 \mathrm{FO}$ was indicated by age $\geq 72$ years [area under the curve (AUC) 0.908 (95\% confidence interval (CI), 0.761-0.979), Se 84.2 (60.4-96.6), Sp 93.7 (69.8-99.8), P<0.001)], GCS $\leq 13$ [AUC 0.661 (95\% CI 0.482-0.812), Se 52.63 (28.9-75.6), Sp 81.25 (54.4-96.0), $\mathrm{P}=0.0598)]$ and $\mathrm{D}$-dimer $>0.905 \mu \mathrm{g} / \mathrm{ml}$ fibrinogen equivalent unit [FEU; AUC 0.845 (95\% CI 0.683-0.945), Se 84.21 (60.4-96.6), Sp 87.50 (61.7-98.4), $\mathrm{P}<0.001]$. The receiver operating characteristic curve of admission D-dimer predicting day $90 \mathrm{FO}$ is presented in Fig. 2.

\section{Discussion}

In this observational cohort of patients with spontaneous $\mathrm{ICH}$, lower admission D-dimer indicated an improved day $90 \mathrm{FO}$ and independence status. Increased age, previous stroke and certain initial imagistic parameters, including IVH, ECV and cerebral atrophy, implied an unfavorable outcome. The results indicated that D-dimer may also anticipate the development of

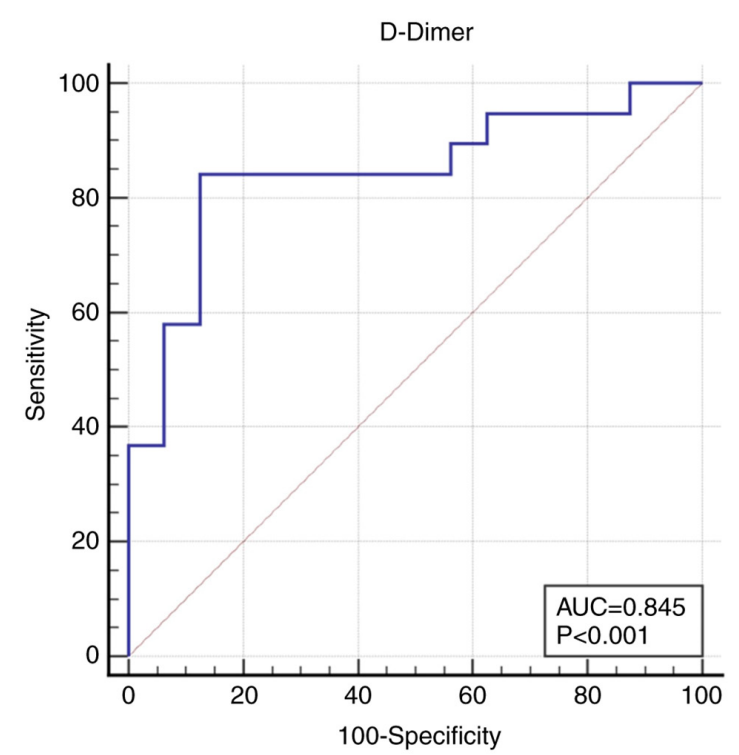

Figure 2. Receiver operation characteristic curve of admission D-dimer predicting day 90 functional outcome. Cut-off, $0.905 \mu \mathrm{g} / \mathrm{ml}$ fibrinogen equivalent units. AUC, area under curve.

ENW, and modestly reflect admission PHE, whereas certain inflammatory markers may correlate with PHE growth.

Our POC results of baseline D-dimer supported previously published data on the use of D-dimer in prognosing day 90 unfavorable FO $(40,41)$, with an admission level of $0.905 \mu \mathrm{g} / \mathrm{ml}$ FEU predicting a poor 3-month outcome with a 
sensitivity and specificity of $\sim 85 \%$. Previous reports identified values $\sim 0.5 \mathrm{mg} / \mathrm{l} \mathrm{FEU}$ as estimating a poor outcome $(41,42)$. Nevertheless, the correlation between D-dimer and 3-month dependence status should be investigated further to verify the results of the present study. The mechanism through which D-dimer impacts the sICH prognostic value is yet to be established, as it is traditionally a hypercoagulability marker and more recently has been considered for potential application in ischemic stroke diagnosis (43). Evidence of elevated D-dimer as expression of increased fibrinolysis, hence contributing to a hypocoagulable status and extensive hemorrhages, is rather scarce $(35,36,44)$. The present study consistently associated D-dimer with clinical endpoints, including admission neurological status expressed as GCS, throughout the entire follow-up period, alongside baseline PHE. Nevertheless, a correlation with ICH volume was not identified in the present study, despite being frequently reported in previous studies $(37,40,41)$. With ICH volume as a known determinant of admission neurological status and ENW $(40,44,45)$, a larger cohort might associate D-dimer with baseline volume and the evolution of neurological status, thus supporting the hypocoagulability theory.

ICH induces a state of systemic and peripheral inflammation, thus increasing circulating WBC and recruiting certain molecules within the affected area, which amplifies local damage $(13,22)$. Previous studies have reported results for several inflammatory biomarkers and calculated indexes (Hb, absolute RDW, GRA, LYM, PLT, NLR, PLR) that contribute to the existing data on FO prognostication $(5,6,17,19,28,29,46,47)$, but the cohort assessed in the present study did not display statistically significant results, only in-line tendencies with previously published evidence. Furthermore, moderate negative correlations were determined between WBC, GRA, SII and PHE growth. However, these were not consistent with existing theories on the contribution of acute inflammation to PHE enlargement (13), and subsequently to an unfavorable FO $(6,13,15,16)$. This contradiction might reside in the modest sample size of the current analysis and of the fact that the GRA population was reported as a substitute for NEU, the former also including EOS alongside NEU. Recent evidence has shown a significant increase in peripheral WBC, GRA and MON population in patients with acute ICH, whereas the LYM population has decreased (48). The contribution of EOS to SICH prognostication is not completely understood; however, Chen et al (14) correlated EOS count with increased risk of HE. Moreover, the short interval from symptom onset to CBC sampling in the present cohort (39) might have prevented the documentation of the activation of local and systemic inflammation $(13,22,48)$. In the present study, MID was documented as a surrogate for MON, but its values incorporate multiple cell populations. SII is a parameter that is documented in regard to hospital discharge outcome of patients with sICH (20); NEU and LYM reflect inflammation, whilst PLT reflect vascular permeability. As such, SII components could depict local PHE metabolism. The results of the present study were in line with previous data on larger SII values in the unfavorable FO subgroup (20), without reaching statistical significance. Furthermore, there was no association with day 7 neurological status (as a proxy for the reported discharge FO) (20) or day $90 \mathrm{FO}$, which indicated that the timing of the most effective inflammatory panel requires further investigation.

As all reported individual inflammatory markers (WBC, GRA, LYM, MID and CRP) failed to predict day 90 FO, the analysis on calculated indexes (NLR, LMR, PLR and SII) produced similar results. Subsequently, further extensive research is required to validate whether such POC derived indexes can impact outcome prognostication in a similar manner as previous evidence has indicated $(5,12,17,46)$.

At present, the results regarding CRP are inconclusive, despite existing evidence of its association with $\mathrm{HG}$, ENW, mortality and 3-month outcome $(5,7,9,24)$, including in-hospital mortality data reported on a consistent ED-based cohort (9). Currently, hs-CRP assays can only measure plasma pentameric CRP, thus failing to incorporate the extent of the neuroinflammatory response to ICH (24). Furthermore, a pre-existing subliminal inflammatory status, though not detected as an infection or chronic inflammation, might affect the coagulation function and the vessel wall pathophysiology, contributing to persistent vessel leakage.

PLT and PLR are well-known indicators of mortality $(8,49)$ and increase the chances of a negative day 90 FO (6,17-19). Although a similar $25 \%$ of each study group was under antiplatelet medication when sICH occurred, the present analysis only documented a moderate negative correlation of PLT with admission ICH volume and day 7 neurological status, in spite of previous discussions on its implications within the existing inflammatory process accompanying sICH (19). Nevertheless, Zhang and Shen (19) demonstrated that ICU rather than ED admission PLR values are relevant for outcome estimation.

In regard to $\mathrm{Hb}$ levels, lower mean admission values were recorded in the unfavorable outcome group, without statistical significance or without meeting the criteria of the definition of anemia (39). RDW is another inexpensive automatically generated hematology parameter that is impacted by inflammation (29), and our results indicated a modest correlation with admission PHE, but this was not associated with day 90 FO.

If sICH is considered as having a systemic impact, then metabolic and cardiac biomarkers could indicate its amplitude. However, random admission hyperglycemia did not reflect the severity of sICH (50), nor was it associated with day $90 \mathrm{FO}$ estimation (6), in spite of the modest negative correlation with PHE growth. Mean admission cTnI levels did not differ among outcome groups and the analysis conducted in the present study did not correlate this parameter with any of the study endpoints. Previous studies on troponin levels have concluded that peak serum cTnI values rather than admission values reflect a poor outcome, including mortality $(33,34)$. Therefore, we speculate that the results of the present study suggest further investigating POC testing.

To the best of our knowledge, the present study was one of the few studies on POC testing in acute sICH within an $\mathrm{ED}$, yet its value is limited due to its one-center location and modest sample size, meaning that the current results require further investigation in order to be validated in sICH management. As the sICH population is of an increasing age, D-dimer interpretation should be considered cautiously. The heterogeneity of control CT scan acquisition is another restraint, as the timing of the second scan varied greatly 
and, as such, ICH and PHE progression were not reflected uniformly. Therefore, serial sampling of inflammatory biomarkers and a more restrictive protocol for control scans might identify the most relevant time point for a predictive inflammatory panel.

In conclusion, several biomarkers showed modest correlations with the progression of $\mathrm{sICH}$ and the day $90 \mathrm{FO}$, advocating for extensive research on the contribution of ED POC routine biomarkers as outcome assessment tools in hemorrhagic stroke. D-dimer could be a promising maker, as lower admission values could indicate an improved day 90 FO and anticipate the development of PHE growth and ENW. The predictive utility of D-dimer on independence status is a novelty at the present moment and further research is needed to validate the current observations in the acute care setting.

\section{Acknowledgements}

Not applicable.

\section{Funding}

The present study was supported by the 'Iuliu Hațieganu' University of Medicine and Pharmacy Cluj-Napoca via an internal $\mathrm{PhD}$ research program (grant no. 7690/74/15.04.2016 and PCD no. 5200/64/01.03.2017).

\section{Availability of data and materials}

The datasets used and/or analyzed during the current study are available from the corresponding author on reasonable request.

\section{Authors' contributions}

EMM, AG and LPD conceptualized the study. EMM, AG, ML, $\mathrm{CC}$ and LPD performed the experiments. Formal analysis was conducted by EMM and SCV. Investigations were performed by EMM, AG, ML, CC and LPD. Resources were accrued by EMM, AG and LPD. The original draft preparation was carried out by EMM. Reviewing and editing of the manuscript were performed by EMM, AG, SCV, ML, CC and LPD. AG and LPD provided supervision. EMM and AG confirm the authenticity of all the raw data. All authors have read and approved the final version of the manuscript.

\section{Ethics approval and consent to participate}

The present study was conducted according to the Declaration of Helsinki and was approved by the Ethics Committee of the 'Iuliu Hațieganu' University of Medicine and Pharmacy (approval no. 441/24.11.2016). All patients or legal representatives provided written informed consent.

\section{Patient consent for publication}

Not applicable.

\section{Competing interests}

The authors declare that they have no competing interests.

\section{References}

1. Krishnamurthi RV, Ikeda T and Feigin VL: Global, regional and country-specific burden of ischaemic stroke, intracerebral haemorrhage and subarachnoid haemorrhage: A systematic analysis of the global burden of disease study 2017. Neuroepidemiology 54: 171-179, 2020.

2. An SJ, Kim TJ and Yoon BW: Epidemiology, risk factors, and clinical features of intracerebral hemorrhage: An update. J Stroke 19: 3-10, 2017.

3. McGurgan IJ, Ziai WC, Werring DJ, Al-Shahi Salman R and Parry-Jones AR: Acute intracerebral haemorrhage: Diagnosis and management. Pract Neurol 21: 128-136, 2020.

4. Romanian Ministry of Health: Priority action regarding the treatment of acute stroke. Standard operational procedure regarding patient referral and therapeutic protocol. Romanian Ministry of Health, Bucharest, 2015. https://www.neurology.ro/protocoale-si -ghiduri-meniu-main/protocol-avc. Accessed June 28, 2021.

5. Saand AR, Yu F, Chen J and Chou SH: Systemic inflammation in hemorrhagic strokes-a novel neurological sign and therapeutic target? J Cereb Blood Flow Metab 39: 959-988, 2019.

6. Fonseca S, Costa F, Seabra M, Dias R, Soares A, DIas C, Azevedo E and Castro P: Systemic inflammation status at admission affects the outcome of intracerebral hemorrhage by increasing perihematomal edema but not the hematoma growth. Acta Neurol Belg 121: 649-659, 2021.

7. Di Napoli M, Parry-Jones AR, Smith CJ, Hopkins SJ, Slevin M, Masotti L, Campi V, Singh P, Papa F, Popa-Wagner A, et al: $\mathrm{C}$-reactive protein predicts hematoma growth in intracerebral hemorrhage. Stroke 45: 59-65, 2014.

8. Kim Y, Han MH, Kim CH, Kim JM, Cheong JH and Ryu JI: Increased short-term mortality in patients with spontaneous intracerebral hemorrhage and its association with admission glucose levels and leukocytosis. World Neurosurg 98: 503-511, 2017.

9. Yoshinaga R, Doi Y, Ayukawa K and Ishikawa S: High-sensitivity $C$ reactive protein as a predictor of inhospital mortality in patients with cardiovascular disease at an emergency department: A retrospective cohort study. BMJ Open 7: e015112, 2017.

10. Tao C, Hu X, Wang J, Ma J, Li H and You C: Admission neutrophil count and neutrophil to lymphocyte ratio predict 90-day outcome in intracerebral hemorrhage. Biomark Med 11: 33-42, 2017.

11. Yu S, Arima H, Heeley E, Delcourt C, Krause M, Peng B, Yang J, Wu G, Chen X, Chalmer J, et al: White blood cell count and clinical outcomes after intracerebral hemorrhage: The INTERACT2 trial. J Neurol Sci 361: 112-116, 2016.

12. Qi H, Wang D, Deng X and Pang X: Lymphocyte-to-monocyte ratio is an independent predictor for neurological deterioration and 90-day mortality in spontaneous intracerebral hemorrhage. Med Sci Monit 24: 9282-9291, 2018.

13. Urday S, Kimberly WT, Beslow LA, Vortmeyer AO, Selim MH, Rosand J, Simard JM and Sheth KN: Targeting secondary injury in intracerebral haemorrhage-perihaematomal oedema. Nat Rev Neurol 11: 111-122, 2015.

14. Chen Q, Liu J, Xu H, He W, Li Y, Jiao L, Xiang Y, Zhan C, Chen J, Yang X, et al: Association between eosinophilic leukocyte count and hematoma expansion in acute spontaneous intracerebral hemorrhage. Front Neurol 10: 1164, 2019.

15. Urday S, Beslow LA, Dai F, Zhang F, Battey TW, Vashkevich A, Ayres AM, Leasure AC, Selim MH, Simard M, et al: Rate of perihematomal edema expansion predicts outcome after intracerebral hemorrhage. Crit Care Med 44: 790-797, 2016.

16. Grunwald Z, Beslow LA, Urday S, Vashkevich A, Ayres AM, Greenberg SM, Goldstein JN, Leasure A, Shi FD, Kahle KT, et al: Perihematomal edema expansion rates and patient outcomes in deep and lobar intracerebral hemorrhage. Neurocrit Care 26: 205-212, 2017.

17. Zhang W and Shen Y: Platelet-to-lymphocyte ratio as a new predictive index of neurological outcomes in patients with acute intracranial hemorrhage: A retrospective study. Med Sci Monit 24: 4413-4420, 2018.

18. Mayda-Domaç F, Misırlı H and Yilmaz M: Prognostic role of mean platelet volume and platelet count in ischemic and hemorrhagic stroke. J Stroke Cerebrovasc Dis 19: 66-72, 2010.

19. Lin CY, Chang CY, Sun CH, Li TY, Chen LC, Chang ST and Wu YT: Platelet count and early outcome in patients with spontaneous cerebellar hemorrhage: A retrospective study. PLoS One 10: e0119109, 2015. 
20. Trifan G and Testai FD: Systemic immune-inflammation (SII) index predicts poor outcome after spontaneous supratentorial intracerebral hemorrhage. J Stroke Cerebrovasc Dis 29: 105057, 2020.

21. Adeoye O, Walsh K, Woo JG, Haverbusch M, Moomaw CJ, Broderick JP, Kissela BM, Kleindorfer D, Flaherty ML and Woo D: Peripheral monocyte count is associated with case fatality after intracerebral hemorrhage. J Stroke Cerebrovasc Dis 23: e107-e111, 2014

22. Tapia-Pérez JH, Karagianis D, Zilke R, Koufuglou V, Bondar I and Schneider T: Assessment of systemic cellular inflammatory response after spontaneous intracerebral hemorrhage. Clin Neurol Neurosurg 150: 72-79, 2016.

23. Zhang F, Ren Y, Fu W, Yang Z, Wen D, Hu X, Tao C, Li X, You $\mathrm{C}$, Xin T and Yang M: Predictive accuracy of neutrophil-to-lymphocyte ratio on long-term outcome in patients with spontaneous intracerebral hemorrhage. World Neurosurg 125 : e651-e657, 2019.

24. Di Napoli M, Slevin M, Popa-Wagner A, Singh P, Lattanzi S and Divani AA: Monomeric C-reactive protein and cerebral hemorrhage: From bench to bedside. Front Immunol 9: 1921, 2018.

25. Kumar MA, Rost NS, Snider RW, Chanderraj R, Greenberg SM, Smith EE and Rosand J: Anemia and hematoma volume in acute intracerebral hemorrhage. Crit Care Med 37: 1442-1447, 2009.

26. Roh DJ, Albers DJ, Magid-Bernstein J, Doyle K, Hod E, Eisenberger A, Murthy S, Witsch J, Park S, Agarwal S, et al: Low hemoglobin and hematoma expansion after intracerebral hemorrhage. Neurology 93: e372-e380, 2019.

27. Diedler J, Sykora M, Hahn P, Heerlein K, Schölzke MN, Kellert L, Bösel J, Poli S and Steiner T: Low hemoglobin is associated with poor functional outcome after non-traumatic, supratentorial intracerebral hemorrhage. Crit Care 14: R63, 2010.

28. Zhang S, Pan X, Wei C, Wang L, Cheng Y, Hu Z, Dong W, Liu M and $\mathrm{Wu} \mathrm{B}$ : Associations of anemia with outcomes in patients with spontaneous intracerebral hemorrhage: A meta-analysis. Front Neurol 10: 406, 2019.

29. Cui Z, Liu C, Sun G, Huang L and Zhou W: A prognostic nomogram incorporating red cell distribution width for patients with intracerebral hemorrhage. Medicine (Baltimore) 99: e23557, 2020.

30. Hays A and Diringer MN: Elevated troponin levels are associated with higher mortality following intracerebral hemorrhage. Neurology 66: 1330-1334, 2006.

31. Xu M, Lin J, Wang D, Liu M, Hao Z and Lei C: Cardiac troponin and cerebral herniation in acute intracerebral hemorrhage. Brain Behav 7: e00697, 2017.

32. Sandhu R, Aronow WS, Rajdev A, Sukhija R, Amin H, D'aquila K and Sangha A: Relation of cardiac troponin I levels with in-hospital mortality in patients with ischemic stroke intracerebral hemorrhage, and subarachnoid hemorrhage. Am J Cardiol 102: 632-634, 2008.

33. Gerner ST, Auerbeck K, Sprügel MI, Sembill JA, Madžar D, Gölitz P, Hoelter P, Kuramatsu JB, Schwab S and Huttner HB: Peak troponin I levels are associated with functional outcome in intracerebral hemorrhage. Cerebrovasc Dis 46: 72-81, 2018.

34. He Y, Liu Q, Wang J, Wang DW, Ding H and Wang W: Prognostic value of elevated cardiac troponin I in patients with intracerebra hemorrhage. Clin Cardiol 43: 338-345, 2020.

35. Zhou Z, Liang Y, Zhang X, Xu J, Kang K, Qu H, Zhao C and Zhao M: Plasma D-dimer concentrations and risk of intracerebral hemorrhage: A systematic review and meta-analysis. Front Neurol 9: 1114, 2018.

36. Di Castelnuovo A, Agnoli C, de Curtis A, Giurdanella MC, Sieri S, Mattiello A, Matullo G, Panico S, Sacerdote C, Tumino R, et al: Elevated levels of D-dimers increase the risk of ischaemic and haemorrhagic stroke. Findings from the EPICOR Study. Thromb Haemost 112: 941-946, 2014.
37. Cheng X, Zhang L, Xie NC, Ma YQ and Lian YJ: High plasma levels of D-dimer are independently associated with a heightened risk of deep vein thrombosis in patients with intracerebral hemorrhage. Mol Neurobiol 53: 5671-5678, 2016.

38. Bustamante A, López-Cancio E, Pich S, Penalba A, Giralt D, García-Berrocoso T, Ferrer-Costa C, Gasull T, Hernández-Pérez M, Millan M, et al: Blood biomarkers for the early diagnosis of stroke: The stroke-chip study. Stroke 48: 2419-2425, 2017

39. Mureșan EM, Golea A, Bolboacă S and Perju-Dumbravă L: Feasibility of a pilot study on point-of-care biomarkers in spontaneous intracerebral hemorrhage in an emergency setting. Med Pharm Rep 94: 307-317, 2021.

40. Delgado P, Alvarez-Sabín J, Abilleira S, Santamarina E, Purroy F, Arenillas JF, Molina CA, Fernández-Cadenas I, Rosell A and Montaner J: Plasma d-dimer predicts poor outcome after acute intracerebral hemorrhage. Neurology 67: 94-98, 2006.

41. Hu X, Fang Y, Ye F, Lin S, Li H, You C and Liu M: Effects of plasma D-dimer levels on early mortality and long-term functional outcome after spontaneous intracerebral hemorrhage. J Clin Neurosci 21: 1364-1367, 2014.

42. Zhou Q, Zhang D, Chen X, Yang Z, Liu Z, Wei B, Jin M, Feng K, Guo C, Sun J, et al: Plasma D-dimer predicts poor outcome and mortality after spontaneous intracerebral hemorrhage. Brain Behav 11: 462-468, 2021.

43. Misra S, Montaner J, Ramiro L, Arora R, Talwar P, Nath M, Kumar A, Kumar P,Pandit AK, Mohania D, et al: Blood biomarkers for the diagnosis and differentiation of stroke: A systematic review and meta-analysis. Int J Stroke 15: 704-721, 2020.

44. Cho TG, Lee JC, Park SW, Chung C, Nam TK and Hwang SN Relationship between systemic thrombogenic or thrombolytic indices and acute increase of spontaneous intracerebral hemorrhage. J Cerebrovasc Endovasc Neurosurg 16: 159-165, 2014.

45. Specogna AV, Turin TC, Patten SB and Hill MD: Factors associated with early deterioration after spontaneous intracerebral hemorrhage: A systematic review and meta-analysis. PLoS One 9: e96743, 2014.

46. Lattanzi S, Brigo F, Trinka E, Cagnetti C, Di Napoli M and Silvestrini M: Neutrophil-to-lymphocyte ratio in acute cerebral hemorrhage: A system review. Transl Stroke Res 10: 137-145, 2019.

47. Liu S, Liu X, Chen S, Xiao Y and Zhuang W: Neutrophil-lymphocyte ratio predicts the outcome of intracerebral hemorrhage: A meta-analysis. Medicine (Baltimore) 98: e16211, 2019

48. Jiang C, Wang Y, Hu Q, Shou J, Zhu L, Tian N, Sun L, Luo H, Zuo F, Li F, et al: Immune changes in peripheral blood and hematoma of patients with intracerebral hemorrhage. FASEB J 34: 2774-2791, 2020

49. Zou Y, Zhang W, Huang C and Zhu Y: Clinical significance of neutrophil to lymphocyte ratio and platelet to lymphocyte ratio in acute cerebral hemorrhage with gastrointestinal hemorrhage, and logistic regression analysis of risk factors. Exp Ther Med 18: 1522-1538, 2019.

50. Zhao Y, Yang J, Zhao H, Ding Y, Zhou J and Zhang Y: The association between hyperglycemia and the prognosis of acute spontaneous intracerebral hemorrhage. Neurol Res 39: 152-157, 2017.

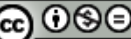

This work is licensed under a Creative Commons Attribution-NonCommercial-NoDerivatives 4.0 International (CC BY-NC-ND 4.0) License. 